Vol. 19(2010): 207-213.

\title{
Comparison of different models for the estimation of genetic parameters of body weight traits in Moghani sheep
}

\author{
Navid Ghavi Hossein-Zadeh*1 and Mehrnaz Ardalan ${ }^{2}$ \\ ${ }^{1}$ Department of Animal Science, Faculty of Agriculture, University of Guilan, PO Box 41635-1314, Rasht, Iran \\ *e-mail: nhosseinzadeh@guilan.ac.ir \\ ${ }^{2}$ Department of Animal Science, University College of Agriculture and Natural Resources, \\ University of Tehran, PO Box 31587-77871, Karaj, Iran
}

\begin{abstract}
Genetic parameters for birth weight (BW), 3-month weight (3MW), 6-month weight (6MW), 9-month weight $(9 \mathrm{MW})$ and yearling weight $(\mathrm{YW})$ in Iranian Moghani sheep were estimated using restricted maximum likelihood procedure of MTDFREML program. The data and pedigree information used in this research were collected at the Breeding station of Moghani sheep (Ardebil province, Iran) during 1987-2005. Six different animal models were fitted, differentiated by including or excluding maternal effects, with and without covariance between maternal and direct genetic effects. The estimates for direct heritability ranged from 0.31 to $0.54,0.21$ to $0.34,0.13$ to $0.25,0.11$ to 0.22 and 0.10 to 0.17 for $\mathrm{BW}, 3 \mathrm{MW}, 6 \mathrm{MW}, 9 \mathrm{MW}$ and $\mathrm{YW}$, respectively. The estimates were substantially higher when maternal effects, either genetic or environmental, were ignored in the model. The results of this study show that full models with maternal genetic and environmental effects gave the most accurate estimates for body weight traits in Moghani sheep.
\end{abstract}

Key-words: heritability, animal model, growth traits, maternal effect, meat sheep

(C) Agricultural and Food Science

Manuscript received January 2010 


\section{Hossein-Zadeh, N. et al. Estimation of genetic parameters for Moghani sheep}

\section{Introduction}

Sheep production constitutes the most important part of Iranian livestock industry and a total of approximately 50 million heads of sheep of more than twenty breeds and sub-breeds are produced in Iran. Sheep contribute more than $42 \%$ of about 293 million $\mathrm{kg}$ annual meat production in Iran (Vatankhah et al. 2004). Moghani sheep, numbering about 5.5 million, is one of the most important meat breeds among Iranian sheep. They are well-known for their large size, tolerance to weather changes and capability to produce heavy lambs (Nosrati 1998). The breed is fat-tailed, its coat color is predominantly white and its face, legs and feet are brown. In this breed, both sexes are polled. A breeding station was established in 1952 in Ardebil province of Iran in order to genetically improve the performance of Moghani sheep, through recognizing the best rams and distributing them to the commercial flocks. There was a controlled mating system so that the identity of sire and dam of each lamb was known. Ewe lambs and ram lambs were bred at 18 months of age. Rams were used for only one year and ewes may be used for up to 6 years. Lambs were weaned at 120 days of age. Male and female lambs were herded in separate flocks from 6 months of age onwards. Selection took place in July and was primarily based on general appearance and color. Body weight at birth, weaning and at higher ages were sometimes considered as a criteria in selection of replacements.

Early growth of farm animals is determined not only by an animal's genetic potential for growth but also by maternal genetic and permanent environ- mental effects. Hence, to achieve optimum genetic progress in a selection program both the direct and maternal genetic components should be taken into account, especially if there is an antagonistic relationship between them. Studies on various sheep breeds have shown that both direct and maternal genetic influences are important for lamb growth (e.g. Maria et al. 1993, Ligda et al. 2000). Using appropriate models which include dam and other environmental effects can result in a more accurate estimation of variance components. The objective of this study was to estimate genetic parameters for body weight traits in the Moghani sheep, using models with or without maternal genetic and environmental effects.

\section{Material and methods}

\section{Data set}

The data set and pedigree information used in this study were obtained from the breeding station of Moghani sheep (in Ardebil province, Iran) from 1987 to 2005. But larger proportion of data was distributed from 1995 to 2005. The data were screened several times and defective and doubtful data were deleted (e.g., lambs with incomplete records of parentage or with registration numbers lower than the numbers of their parents were left out). The included traits were: Birth weight (BW), 3-month weight (3MW), 6-month weight (6MW), 9-month weight (9MW) and yearling weight (YW). The characteristics of data used in the analyses are presented in Table 1. Singletons, twins

Table 1. Characteristics of data set for Iranian Moghani sheep

\begin{tabular}{lcccc}
\hline Trait & No. of records & Mean $(\mathrm{kg})$ & SD $(\mathrm{kg})$ & CV $(\%)$ \\
\hline BW & 4208 & 4.55 & 0.77 & 16.81 \\
3MW & 4175 & 23.40 & 5.48 & 21.85 \\
6MW & 3138 & 34.24 & 6.31 & 18.44 \\
9MW & 2244 & 38.36 & 9.92 & 15.59 \\
YW & 1342 & 39.28 & 6.99 & 17.79 \\
\hline
\end{tabular}

$\mathrm{BW}=$ Birth weight; 3MW= 3-month weight; $6 \mathrm{MW}=6$-month weight; $9 \mathrm{MW}=9$-month weight; $\mathrm{YW}=$ Yearling weight; $\mathrm{SD}=\mathrm{Standard}$ deviation; $\mathrm{CV}=$ Coefficient of variation 
Vol. 19(2010): 207-213.

and triplets were consisted $63 \%, 36 \%$ and $1 \%$ of all lambings, respectively. Months of birth were grouped into four seasons: January through March (winter), April through June (spring), July through September (summer) and October through December (fall). The results of previous study (Ghavi Hossein-Zadeh 2010) indicated that direct genetic trends were positive and significant $(p<0.05)$ for body weights at different ages in Moghani sheep over the years. Also, the number of animals (in total), inbred animals, grand sires, grand dams, sires, dams, progenies, base animals and non base animals in the pedigree of Moghani sheep were 8494, 1060, 256, 1157, 625, 2828, 6659, 1835 and 6659 , respectively.

\section{Statistical models of genetic analysis}

Variance components and genetic parameters were estimated for each trait using the MTDFREML program (Boldman et al. 1995) by fitting six singletrait animal models that ignore or include additive maternal genetic and permanent environmental effects. The models used to estimate genetic parameters included random effects and fixed effects that were found significant in the general model analyses. Firstly, the GLM procedure (SAS Institute Inc., Cary, NC, 2002) was used for determining the fixed effects that have significant influence on the traits investigated, assuming interactions negligible. Level of significance for the inclusion of effects into the model of analysis was declared at $p<0.05$. The final model included the fixed class effects of year-season (76 levels), sex of lamb and parity of dam, birth type (single, twin, triplet), and the linear covariate effect of age of dam (from 2 through 7 years old). The models used were:

\section{Model 1: $\boldsymbol{y}=\boldsymbol{X} \boldsymbol{b}+\boldsymbol{Z a}+\boldsymbol{e}$}

Model 2: $\boldsymbol{y}=\boldsymbol{X b}+\boldsymbol{Z}_{\boldsymbol{a}} \boldsymbol{a}+{ }_{\boldsymbol{c}} \boldsymbol{c}+\boldsymbol{e}$

Model 3: $\boldsymbol{y}=\boldsymbol{X} \boldsymbol{b}+\boldsymbol{Z}_{\boldsymbol{a}} \boldsymbol{a}+\boldsymbol{Z}_{\boldsymbol{m}} \boldsymbol{m}+\boldsymbol{e}$ with $\operatorname{Co} \mathrm{v}(\boldsymbol{a}, \boldsymbol{m})=0$

Model 4: $\boldsymbol{y}=\boldsymbol{X} \boldsymbol{b}+\boldsymbol{Z}_{\boldsymbol{a}} \boldsymbol{a}+\boldsymbol{Z}_{\boldsymbol{m}} \boldsymbol{m}+\boldsymbol{e}$ with $\operatorname{Co} \mathrm{v}(\boldsymbol{a}, \boldsymbol{m})=\boldsymbol{A} \sigma_{\mathrm{a} m}$

Model 5: $\boldsymbol{y}=\boldsymbol{X} \boldsymbol{b}+\boldsymbol{Z}_{\boldsymbol{a}} \boldsymbol{a}+\boldsymbol{Z}_{\boldsymbol{m}} \boldsymbol{m}+\boldsymbol{Z}_{\boldsymbol{c}} \boldsymbol{c}+\boldsymbol{e}$ with $\operatorname{Co} \mathrm{v}(\boldsymbol{a}, \boldsymbol{m})=0$

Model 6: $\boldsymbol{y}=\boldsymbol{X} \boldsymbol{b}+\boldsymbol{Z}_{\boldsymbol{a}} \boldsymbol{a}+\boldsymbol{Z}_{\boldsymbol{m}} \boldsymbol{m}+\boldsymbol{Z}_{c} c+\boldsymbol{e}$ with $C o$

$$
\mathrm{v}(\boldsymbol{a}, \boldsymbol{m})=\boldsymbol{A} \sigma_{\mathrm{a} \boldsymbol{m}}
$$

Where $\boldsymbol{y}$ is a vector of observations, $\boldsymbol{b}$ is a vector of fixed effects with incidence matrix $\boldsymbol{X}, \boldsymbol{a} \sim$ $\mathrm{N}\left(0, A \sigma_{a}^{2}\right)$ and $\boldsymbol{m} \sim \mathrm{N}\left(0, A \sigma_{a}^{2}\right)$ are vectors of direct and maternal genetic effects with incidence matrices $\boldsymbol{Z}_{\boldsymbol{a}}$ and $\boldsymbol{Z}_{\boldsymbol{m}}$, respectively, $\mathrm{c} \sim \mathrm{N}\left(0, \boldsymbol{I}_{c} \sigma_{c}^{2}\right)$ is a vector of random maternal permanent environmental effects with incidence matrix $\boldsymbol{Z}_{\boldsymbol{c}}$ and $\boldsymbol{e} \sim \mathrm{N}\left(0, \boldsymbol{I}_{n} \sigma_{e}^{2}\right)$ is a vector of random residual effects. Also, $\sigma_{a}^{2}$ is the direct genetic variance, $\sigma_{m}^{2}$ is the maternal genetic variance, $\sigma_{c}^{2}$ is the maternal permanent environmental variance, $\sigma_{e}^{2}$ is the residual variance, $\boldsymbol{A}$ the additive genetic relationship matrix, and $\boldsymbol{I}_{\boldsymbol{c}}$ and $\boldsymbol{I}_{\boldsymbol{n}}$ are the identity matrices of order equal to the number of maternal permanent environmental effects and the number of records, respectively.

Convergence was considered reached when the simplex variance was less than $10^{-8}$. To ensure that a global maximum was reached, several other rounds of iterations were used using results from the previous round as starting values. When estimates did not change at the third decimal, convergence was assumed. The most suitable model amongst all six models was determined based on likelihood ratio tests for each trait (Meyer 1992). An effect was considered to have a significant effect when its inclusion caused a significant increase in $\log$ likelihood, compared to the model in which it was ignored. When -2 times the difference between the log likelihood was greater than a critical value from a chi square distribution with one degree of freedom, the additional random factor was concluded to have had a significant effect. Significance was tested at the level of $p<0.05$ for all traits.

\section{Results}

As it is expected the number of records decreased with age and this decline in the numbers of observations has been due to the harvest/culling and losses of lambs in different ages. Estimates of direct and maternal heritabilities ( $\mathrm{h}^{2}$ and $h_{m}^{2}$ respectively), maternal permanent environmental variance as a proportion of phenotypic variance $\left(\mathrm{c}^{2}\right)$ and -2 loglikelihood values for body weight traits obtained 


\section{Hossein-Zadeh, N. et al. Estimation of genetic parameters for Moghani sheep}

under different models are shown in Table 2. Fitting either maternal permanent environmental effect (model 2) or maternal genetic effect (model 3) as second random effect resulted in significantly $(p<$ 0.001 ) higher log-likelihood and more accuracy for all traits in comparison to the model which ignored these effects (models 1). Addition of covariance between direct and maternal genetic effect (model 4) did not improve the log-likelihood compared with model 3. Inclusion of maternal permanent environmental effect after maternal genetic effect (model 5) improved the log-likelihood significantly for all traits. However, the addition of covariance between direct and maternal genetic effect to model 5 improved the log-likelihood for 3MW, 6MW, 9MW and YW (model 6). While model 6 was the best model for 3MW, 6MW, 9MW and YW, model 5 was the best for BW, indicating that the covariance between direct and maternal effect was negligible for BW.

Model 1 had the highest estimates of $\mathrm{h}^{2}$ for all traits. Fitting either or both of the maternal effects

Table 2. Estimates of genetic parameters for body weights in Iranian Moghani sheep

\begin{tabular}{|c|c|c|c|c|c|c|}
\hline Trait & Model & $h^{2} \pm \mathrm{SE}$ & $h_{m}^{2} \pm \mathrm{SE}$ & $\mathrm{r}_{\mathrm{am}} \pm \mathrm{SE}$ & $\mathrm{c}^{2} \pm \mathrm{SE}$ & $-2 \log \mathrm{L}$ \\
\hline \multirow[t]{6}{*}{$\overline{\mathrm{BW}}$} & 1 & $0.54 \pm 0.04$ & - & - & - & -366.26 \\
\hline & 2 & $0.39 \pm 0.03$ & - & - & $0.20 \pm 0.04$ & -328.09 \\
\hline & 3 & $0.35 \pm 0.03$ & $0.23 \pm 0.05$ & - & - & -326.14 \\
\hline & 4 & $0.35 \pm 0.04$ & $0.20 \pm 0.03$ & $0.14 \pm 0.04$ & - & -330.26 \\
\hline & 5 & $0.32 \pm 0.03$ & $0.17 \pm 0.04$ & - & $0.05 \pm 0.02$ & -230.29 \\
\hline & 6 & $0.31 \pm 0.04$ & $0.19 \pm 0.03$ & $0.31 \pm 0.07$ & $0.09 \pm 0.03$ & -278.42 \\
\hline \multirow[t]{6}{*}{$3 \mathrm{MW}$} & 1 & $0.34 \pm 0.05$ & - & - & - & 15163.09 \\
\hline & 2 & $0.26 \pm 0.03$ & - & - & $0.08 \pm 0.03$ & 15469.84 \\
\hline & 3 & $0.23 \pm 0.02$ & $0.09 \pm 0.03$ & - & - & 15467.46 \\
\hline & 4 & $0.23 \pm 0.02$ & $0.12 \pm 0.03$ & $-0.09 \pm 0.03$ & - & 15449.75 \\
\hline & 5 & $0.22 \pm 0.03$ & $0.10 \pm 0.03$ & - & $0.04 \pm 0.02$ & 15889.75 \\
\hline & 6 & $0.21 \pm 0.04$ & $0.09 \pm 0.04$ & $-0.23 \pm 0.05$ & $0.04 \pm 0.01$ & 16191.54 \\
\hline \multirow[t]{6}{*}{$6 \mathrm{MW}$} & 1 & $0.25 \pm 0.04$ & - & - & - & 13103.81 \\
\hline & 2 & $0.19 \pm 0.05$ & - & - & $0.09 \pm 0.03$ & 13291.64 \\
\hline & 3 & $0.17 \pm 0.03$ & $0.08 \pm 0.04$ & - & - & 13290.69 \\
\hline & 4 & $0.16 \pm 0.04$ & $0.08 \pm 0.03$ & $-0.22 \pm 0.08$ & - & 13273.61 \\
\hline & 5 & $0.14 \pm 0.05$ & $0.07 \pm 0.04$ & - & $0.03 \pm 0.02$ & 13485.62 \\
\hline & 6 & $0.13 \pm 0.04$ & $0.05 \pm 0.02$ & $-0.28 \pm 0.08$ & $0.03 \pm 0.01$ & 13709.62 \\
\hline \multirow[t]{6}{*}{ 9MW } & 1 & $0.22 \pm 0.04$ & - & - & - & 8064.58 \\
\hline & 2 & $0.20 \pm 0.05$ & - & - & $0.08 \pm 0.03$ & 8263.63 \\
\hline & 3 & $0.17 \pm 0.04$ & $0.06 \pm 0.03$ & - & - & 8262.24 \\
\hline & 4 & $0.13 \pm 0.03$ & $0.07 \pm 0.03$ & $-0.34 \pm 0.09$ & - & 8259.51 \\
\hline & 5 & $0.13 \pm 0.04$ & $0.07 \pm 0.02$ & & $0.03 \pm 0.01$ & 8393.81 \\
\hline & 6 & $0.11 \pm 0.03$ & $0.05 \pm 0.02$ & $-0.39 \pm 0.08$ & $0.03 \pm 0.01$ & 8596.31 \\
\hline \multirow[t]{6}{*}{ YW } & 1 & $0.17 \pm 0.04$ & - & - & - & 5195.88 \\
\hline & 2 & $0.14 \pm 0.05$ & - & - & $0.07 \pm 0.02$ & 5396.40 \\
\hline & 3 & $0.14 \pm 0.04$ & $0.04 \pm 0.02$ & - & - & 5395.90 \\
\hline & 4 & $0.13 \pm 0.04$ & $0.04 \pm 0.01$ & $-0.52 \pm 0.12$ & - & 5392.65 \\
\hline & 5 & $0.13 \pm 0.03$ & $0.04 \pm 0.02$ & - & $0.02 \pm 0.01$ & 5497.83 \\
\hline & 6 & $0.10 \pm 0.05$ & $0.03 \pm 0.01$ & $-0.61 \pm 0.14$ & $0.02 \pm 0.01$ & 5606.52 \\
\hline
\end{tabular}

"BW=Birth weight; 3MW= 3-month weight; 6MW=6-month weight; 9MW=9-month weight; $\mathrm{YW}=$ Yearling weight; $h^{2}=$ direct heritability; $h_{m}^{2}=$ maternal heritability; $\mathrm{r}_{\mathrm{m}}=$ correlation between direct and maternal genetic effects; $\mathrm{c}^{2}=$ ratio of maternal permanent environmental effect; $-2 \log \mathrm{L}=-2$ times the log likelihood 
Vol. 19(2010): 207-213.

reduced the estimates of direct heritability $\left(h^{2}\right)$, while exclusion of maternal permanent environmental effects (models 3 and 4 ) resulted in a higher estimate of maternal heritability $\left(h_{m}^{2}\right)$. Therefore, ignoring maternal permanent environmental effects will lead to the overestimation of $h_{m}^{2}$. Similarly, exclusion of maternal genetic effect will inflate the estimates of direct heritability $\left(\mathrm{h}^{2}\right)$. However, the magnitude of overestimation of direct heritability $\left(\mathrm{h}^{2}\right)$ and especially $h_{m}^{2}$ was much smaller for $3 \mathrm{MW}$, $6 \mathrm{MW}, 9 \mathrm{MW}$ and $\mathrm{YW}$. For all traits, $\mathrm{h}^{2}$ was higher than $h_{m}^{2}$ which was higher than $\mathrm{c}^{2}$. The magnitude of maternal heritabilities and $c^{2}$ declined from birth to yearling.

\section{Discussion}

The heritability estimates of the traits in current study are not very different from the reported heritabilities by most researchers (Fogarty 1995, Sargolzaei 1997, Zamani and Mohammadi 2008). The results of some investigations summarized by Vatankhah et al. (2005) in different Iranian breeds, showed that the range of heritability estimates for body weights at different ages was between very low (about 0.05 ) to high (above 0.5 ). Several factors such as breed of the animal, genetic variation within population, management and environmental conditions, method of estimating parameters, etc., would have affected the differences between estimations.

In the current study, the heritability estimates for BW and 3MW were relatively high, whereas for weights after weaning they were low. The likely reasons for the low values of the estimated heritability of weights after 3MW might reflect the effects of variation in environmental and nutritional conditions of the range, herd movement, harsh climatic conditions and herd management, on the animal performance. The estimates of $h^{2}$ were within the range of published estimates, which ranged from 0.07 (Näsholm and Danell 1996, Tosh and Kemp 1994) to 0.62 (Bahreini Behzadi et al. 2005) for BW, and from 0.067 (Khalili et al. 2002) to 0.59 (Bahreini Behzadi et al. 2005) for 3MW. The magnitude of the maternal heritability estimates of BW (0.19-0.23) in this research was equal to the direct heritability and was within the range of those reported from 0.09 by Snyman et al. (1995) to 0.59 by El Fadilli et al. (2000). Snyman et al. (1995) showed a wide variation of estimated heritabilities for birth weight (0.04-0.39) and weaning weight (0.09-0.52) in their report. Estimates for direct and maternal heritabilities for weaning weight in this study were consistent with the estimates of 0.22 and 0.11 , respectively, reported for Targhee (Hanford et al. 2003) and Rambouillet (Hanford et al. 2005), but were greater than the estimates of 0.16 and 0.08 reported for Columbia (Hanford et al. 2002). Miraei-Ashtiani et al. (2007) reported the direct and maternal heritability estimates of 0.49 and 0.11 for $6 \mathrm{MW}$ in Sangsari sheep. The heritability estimates of 9MW in this study were greater than those of 0.08 reported by Miraei-Ashtiani et al. (2007) in Sangsari lambs but lower than that of 0.59 reported by Snyman et al. (1995) in Afrino sheep. Estimate of direct heritability for YW (0.31) obtained in the present study was within the range from 0.10 (Miraei-Ashtiani et al. 2007) to 0.58 (Snyman et al. 1995).

The heritability estimates of post-weaning weights have also been reported for many breeds of sheep in the range of $0.1-0.45$ for different conditions (Fogarty 1995, Vatankhah et al. 2005). In some investigations these traits are reported to have more direct additive genetic component than the pre-weaning weights (e.g. Notter and Hough 1997, Vatankhah et al. 2005).

Estimates of direct heritability for all traits ranged from 0.10 to 0.39 when maternal effects were taken into account. In contrast, excluding these effects from the models gave estimates ranging from 0.17 to 0.54 . These results indicate that the estimates of direct heritability can be biased if maternal effects, either genetic or permanent environmental, are ignored in animal models. However, the exclusion of maternal genetic effect from the model had the biggest impact on the direct heritability. The $\mathrm{h}^{2}$ of BW in particular was highly overestimated when maternal genetic effect was not fitted $\left(h^{2}=0.53\right)$, compared to the estimate with maternal genetic effect $(0.35)$. Inclusion of mater- 
Hossein-Zadeh, N. et al. Estimation of genetic parameters for Moghani sheep

nal permanent environmental effect after maternal genetic effect reduced the direct heritability further to 0.32 . This result is consistent with other reports in the literature (Vaez-Torshizi et al. 1996, Ligda et al. 2000, Edriss et al. 2002). Snyman et al. (1995) reported that ignoring maternal effects, if these effects have a significant influence, leads to the overestimation of direct as well as total heritabilities.

For all traits, the ratios of maternal permanent environmental to phenotypic variances $\left(\mathrm{c}^{2}\right)$ were estimated to be lower than direct heritability in different models. This finding is consistent with the results of several other studies (Ligda et al. 2000, Duguma et al. 2002, Edriss et al. 2002). Snyman et al. (1995) and Neser et al. (2001) ascribed the maternal permanent environmental effect to the influence of the uterus and the effect of multiple births. Relatively small $\mathrm{c}^{2}$ estimates for $3 \mathrm{MW}$ and post-weaning weights most probably reflected differences in rearing abilities of dams that might be influenced by environmental fluctuations between years or their birth/weaning status. Also, the decline in both $h_{m}^{2}$ and $\mathrm{c}^{2}$ is most probably due to increase in temporary environmental or residual effects on lamb's body growth rate (Snyman et al. 1995). Despite the positive estimates of direct and maternal genetic correlation (ram) for BW in Moghani sheep, these estimates were negative for after weaning weights. A negative estimate of the direct and maternal genetic correlation has been observed mostly in field data while it has largely been absent in experimental data sets (Meyer 1997). This could be attributed to factors like more uniform management and lack of preferential treatment in experiments. Alternatively, it may also reflect better identification of contemporary or management groups (Meyer 1997). The negative estimates of the direct and maternal genetic correlations could be affected by small data sets (El Fadili et al. 2000, Al-Shorepy 2001), poor pedigree structure or models fitted for estimation of both direct and maternal heritabilities and the genetic correlations between animal effects (Lee et al. 2000). The antagonism between the impact of an individual's own genes for growth and those of its dam may also be due to natural selection for an intermediate optimum (Tosh and Kemp 1994).

\section{Conclusions}

Estimation of the genetic parameters, based on the best model of analysis, for body weight traits of Moghani sheep is needed for planning an efficient breeding system and development of effective genetic evaluation for this breed of sheep. Ignoring maternal effects (genetic and environmental) led to overestimation of $h^{2}$. Likewise, exclusion of maternal permanent environmental effects resulted in overestimation of $h_{m}^{2}$, particularly for BW. Therefore, in addition to the direct additive genetic effect, maternal genetic and environmental effects need to be considered when carrying out genetic evaluation of growth traits in Moghani sheep.

\section{References}

Al-Shorepy, S.A. 2001. Estimates of genetic parameters for direct and maternal effects on birth weight of local sheep in United Arab Emirates. Small Ruminant Research 39: 219-224.

Bahreini Behzadi, M.R., Eftekhar-Shahroudi, F. \& Van Vleck, L.D. 2005. The study of maternal effects on estimation of heritabilities and determination of environmental factors on early growth traits in Kermani sheep. Journal of Science and Technology in Agriculture and Natural Resources 9: 195-202.

Boldman, K.G., Kriese, L.A., Van Vleck, L.D., Van Tassell, C.P. \& Kachman, S.D. 1995. A Manual for Use of MTDFREML. A Set of Programs to Obtain Estimates of Variances and Covariances [DRAFT]. U.S. Department of Agriculture, Agricultural Research Service, Clay Center, NE, USA.

Duguma, G., Schoeman, S.J., Cloete, S.W.P. \& Jordaan, G.F. 2002. Genetic parameter estimates of early growth traits in the Tygerhoek Merino flock. South African Journal of Animal Science 32: 66-75.

Edriss, M.A., Tahmasebi, F. \& Vatan-Khah, M. 2002. Estimation of variance components for pre-weaning weights in Bakhtiari sheep breed. Proceedings of 7 th World Congress on Genetics Applied to Livestock Production, Montpellier, France.

El Fadilli, M., Michaux, C., Detilleux, J. \& Leroy, P.L. 2000. Genetic parameters for growth traits of the Moroccan Timahdit breed of sheep. Small Ruminant Research 37: 203-208.

Fogarty, N.M. 1995. Genetic parameters for live weight, fat and muscle measurements, wool production and reproduction in sheep. A review. Animal Breeding Abstract 63: 101-143. 


\section{AGRICULTURAL AND FOOD SCIENCE}

Vol. 19(2010): 207-213.

Ghavi Hossein-Zadeh, N. 2010. Estimates of genetic trends for body weight traits of Moghani sheep obtained by a multivariate animal model analysis. ADSA-PSA-AMPACSAS-ASAS congress. Denver, Colorado, USA.

Hanford, K.J., Van Vleck, L.D. \& Snowder, G.D. 2002. Estimates of genetic parameters and genetic change for reproduction, weight, and wool characteristic of Columbia sheep. Journal of Animal Science 80: 3086-3098.

Hanford, K.J., Van Vleck, L.D. \& Snowder, G.D. 2003. Estimates of genetic parameters and genetic change for reproduction, weight, and wool characteristics of Targhee sheep. Journal of Animal Science 81: 630-640.

Hanford, K.J., Van Vleck, L.D. \& Snowder, G.D. 2005. Estimates of genetic parameters and genetic change for reproduction, weight, and wool characteristic of Rambouillet sheep. Small Ruminant Research 57: 175-186.

Khalili, D., Vaez-Torshizi, R., Miraei Ashtiani, R. \& Shoorideh, A. 2002. Estimation of genetic parameters for productive and composite reproductive traits for Iranian Baluchi sheep, using single trait animal model. Proceedings of The First Seminar on Genetic and Breeding Applied to Livestock, Poultry and Aquatics, University of Tehran, Iran, pp. 225-231.

Lee, J.W., Choi, S.B., Jung, Y.H., Keown, J.F. \& Van Vleck, L.D. 2000. Parameter estimates for direct and maternal genetic effects on yearling, eighteen-month, and slaughter weights of Korean native cattle. Journal of Animal Science 78: 1414-1421.

Ligda, C., Gabriilidis, G., Papadopoulos, T. \& Georgoudis, A. 2000. Investigation of direct and maternal genetic effects on birth and weaning weight of Chios lambs. Livestock Production Science 67: 75-80.

Maria, G.A., Boldman, K.G. \& Van Vleck, L.D. 1993. Estimates of variances due to direct and maternal effects for growth traits of Romanov sheep. Journal of Animal Science 71: 845-849.

Meyer, K. 1992. Variance components due to direct and maternal effect for growth traits in Australian beef cattle. Livestock Production Science 31: 179-203.

Meyer, K. 1997. Estimates of genetic parameters for weaning weight of beef cattle accounting for directmaternal environmental co variances. Livestock Production Science 52: 187-199.

Miraei-Ashtiani, S.R., Seyedalian, S.A.R. \& Moradi Shahrbabak, M. 2007. Variance components and heritabilities for body weight traits in Sangsari sheep, using univariate and multivariate animal models. Small Ruminant Research 73: 109-114.
Näsholm, A. \& Danell, O. 1996. Genetic relationships of lamb weight, maternal ability, and mature ewe weight in Swedish finewool sheep. Journal of Animal Science 74: 329-339.

Neser, F.W.C., Erasmus, G.J. \& VanWyk, J.B. 2001. Genetic parameter estimates for pre-weaning weight traits in Dorper sheep. Small Ruminant Research 40: 197-202.

Nosrati, M. 1998. Estimation of genetic and phenotypic parameters for body weight at different ages and yearly wool production in Moghani sheep. M.Sc. Thesis. University of Tabriz, Iran.

Notter, D.R. \& Hough, J.D. 1997. Genetic parameter estimates for growth and fleece characteristics in Targhee sheep. Journal of Animal Science 75: 1729-1737.

Sargolzaei, M. 1997. Genetic and environment trend for some production traits in Lori-Bakhtiari Sheep. M.Sc. Thesis. Faculty of Agriculture, Isfahan University of Technology, Isfahan, Iran.

SAS 2002. SAS User's guide v. 9.1: Statistics. SAS Institute, Inc, Cary, NC.

Snyman, M.A., Erasmus, G.J., van Wyk, J.B. \& Olivier, J.J. 1995. Direct and maternal (co) variance components and heritability estimates for body weight at different ages and fleece traits in Afrino sheep. Livestock Production Science 44: 229-235.

Tosh, J.J. \& Kemp, R.A. 1994. Estimation of variance components for lamb weights in three sheep populations. Journal of Animal Science 72: 1184-1190.

Vaez-Torshizi, R.V., Nicholas, F.W. \& Raadsma, H.W. 1996. REML estimates of variance and covariance components for production traits in Australian Merino sheep, using an animal model. 1. Body weight from birth to 22 months. Australian Journal of Agricultural Research 47: 1235-1249.

Vatankhah, M., Moradi Sharebabak, M., Nejati Javarami, A., Miraei-Ashtiani, S.R. \& Vaez Torshizi, R. 2004. A review of sheep breeding in Iran. Proc. 1st Iranian Congr. Anim. Aqua. Sci., Tehran, Iran: 591-597.

Vatankhah, M., Moradi Shahrbabak, M., Nejati-Javaremi, A., Miraei-Ashtiani, S.R. \& Vaez Torshizi, R. 2005. A review of growth traits parameters estimation for some Iranian breeds of sheep. Pajuhesh and Sazandegi 69: 19-28.

Zamani, P. \& Mohammadi, H. 2008. Comparison of different models for estimation of genetic parameters of early growth traits in the Mehraban sheep. Journal of Animal Breeding and Genetics 125: 29-34. 